\title{
Modulatory Effects of Gut Microbiota on Constipation: The Commercial Beverage Yakult Shapes Stool Consistency
}

TO THE EDITOR: It is becoming increasingly recognized that the presence of healthy and diverse gut microbiota is important to achieve or maintain a regular normal bowel movement. It is well known that patients with constipation could be expected to alleviation from the symptoms by the intake of fermented foods such as yogurt, kimchi, and sauerkraut. ${ }^{1}$ Lactobacillus and Bifidobacterium are especially known to support digestive health by generating short-chain fatty acids (SCFAs). The major SCFA in humans are butyrate, propionate, and acetate, where butyrate is the major energy source for colonocytes, propionate is destined for uptake by the liver, and acetate enters the peripheral circulation to be metabolized by peripheral tissues. ${ }^{2}$

Dietary supplementation with fibers, such as oligofructose, oat bran, and apple pectin, is known to promote a significant production of SCFA upon fermentation by saccharolytic bacteria (capable of hydrolyzing or otherwise breaking down a sugar molecule) in the gut lumen. ${ }^{3}$ Therefore, there are at least two common ways proposed to increase gut SCFA ie, probiotics (live bacteria such as yogurt containing Lactobacillus and Bifidobacterium) and prebiotics (fiber fermented food containing supplement for bacteria), as well synbiotics (mixtures of prebiotics and probiotics).

Yakult is a dairy beverage that contains Yakult's exclusive probiotic Lactobacillus casei strain Shirota ( $\mathrm{LcS}$ ), discovered by a Japanese scientist Dr. Minoru Shirota in 1930. Daily consumption of Yakult helps improve digestion and helps build immunity. ${ }^{4}$ Chen et $\mathrm{al}^{5}$ recently published a study "Differential effects of Lactobacillus casei strain Shirota on patients with constipation regarding stool Consistency in China."

The study performed by Chen et al ${ }^{5}$ was based on a subjective survey and a series of laboratory analyses, without comparative design (placebo-controlled, double-blind, parallel-group). Sixtytwo adults who had constipation were recruited and divided into 3 groups upon stool consistency classification, ie, hard stool (HS), normal stool (NS), soft stool (SS). A 4-week consumption of Yakult
(100 mL containing $1 \times 10^{10}$ living $\mathrm{LcS}$ cells) was evaluated for the efficacy of constipation symptoms, defecation frequency, stool SCFA concentrations, abundancy of the intestinal microbiota and the intestinal environment of each subgroup were evaluated.

The survey shows all the constipation symptoms statistically significant improved conclusively in favor of Yakult beverage in improving all constipation symptoms. Especially, the stool quality improvement is featured in both watery and hard stools which all shifted to a normal consistency when compared before and after Yakult intervention.

Stool SCFA levels, measured by gas chromatography, were shown a sequential distribution as SS > NS > HS in the baseline. Differently, after the ingestion of Yakult, the levels of SCFA were elevated only in HS $(35.1 \%$ in acetate, $36.8 \%$ in propionate, and $39.7 \%$ in butyrate). Whereas, the levels of SCFA were decreased only in SS after ingestion of Yakult (28.3\% in acetate, $19.3 \%$ in propionate and $18.6 \%$ in butyrate).

Bacterial 16S ribosomal RNA V3-V4 region was analyzed by using Illumina MiSeq Sequencing in comparison of the abundances of different genera among 3 subgroups. Yakult, compared to baseline, increased the abundances of Pseudobutyrivibrio and Roseburia in HS and decreased the Pseudobutyrivibrio abundance in SS, and decreased Firmicutes in all patients.

This is enriched informative research, however, there are some concerns on the article and the data raised as below:

Firstly, part of this study was a subjective survey and the Yakult probiotic effect in constipation was well known. Therefore, the "true pharmacological effect" was inevitably acknowledged to all participants including observers and observees, which may introduce a bias into the study. Secondly, all recruited subjects were aged 18-45 with the best range of body mass index (Table 1) indicating participants generally in a healthier population category. In other words, this current study was excluded from the highest risk constipation populations such as the elderly, children and obese. Thus, caution is 
urged for participants who may not be in true constipation, hence, the conceit of assuming that any intervention of gut bacteria has strong "healing" powers may have existed in the study.

Not much information on the subjects provided in the article leads the audience to be suspicious of whether the participants were healthier, occasional constipation, constipation-predominant irritable bowel syndrome, etc. It is indicated that the "diagnosis" was from a qualified hospital, however, no accountable evidence supports those participants who were constipation patients, no constipation history (length) and no pelvic floor condition was provided. Adversely, as seen in Table 2, an average of about 4 times/week defecation frequency was diagnosed as "functional constipation," which is unfulfilled with Rome III criteria. ${ }^{6}$ Here, we have to restate functional constipation that defecation frequency occurs less than 3 times per week for a 3 months' period.

Stool consistency is what focused in this article as seen in Table 3, which shows the effect of drinking Yakult on stool consistency: kept on "normal" in NS, changed toward "normal" in HS, and reversed "loose" to "normal" in SS. However, there is a difference when comparing the calculations, wherein Table 2 there was an average

Table 1. Subject Demographics (Adapted From Chen et $\mathrm{al}^{5}$ )

\begin{tabular}{|c|c|c|c|c|}
\hline \multirow{2}{*}{ Variable } & \multicolumn{3}{|c|}{ BSFS } & \multirow{2}{*}{$P$-value } \\
\hline & $\mathrm{HS}(\mathrm{n}=23)$ & $\mathrm{NS}(\mathrm{n}=27)$ & $\mathrm{SS}(\mathrm{n}=12)$ & \\
\hline Sex (\% of females) & 83 & 85 & 100 & $>0.999^{\mathrm{a}}$ \\
\hline Age (yr) & $27.5(10.8)$ & $24.0(7.0)$ & $23.0(3.8)$ & $0.007^{\mathrm{b}}$ \\
\hline Height (m) & $1.63(0.13)$ & $1.63(0.10)$ & $1.64(0.06)$ & $0.851^{\mathrm{b}}$ \\
\hline Weight (kg) & $57.5(7.8)$ & $55.0(15.0)$ & $52.5(8.3)$ & $0.503^{\mathrm{b}}$ \\
\hline $\mathrm{BMI}\left(\mathrm{kg} / \mathrm{m}^{2}\right)$ & $21.0(2.4)$ & $20.7(3.5)$ & $19.6(1.7)$ & $0.448^{\mathrm{b}}$ \\
\hline
\end{tabular}

BSFS, Bristol Stool Form Scale; HS, hard stool; NS, normal stool; SS, soft stool; BMI, body mass index.

$P$-value was calculated by ${ }^{\mathrm{a}} \mathrm{Chi}$-squared test and ${ }^{\mathrm{b}} \mathrm{Kruskal}$ Wallis test.

Data are presented as median (interquartile range).

Table 2. Effects of Lactobacillus casei Strain Shirota on Constipation Symptoms and Defecation Frequency (Adapted From Chen et al ${ }^{5}$ )

\begin{tabular}{lcccc}
\hline \multicolumn{1}{c}{ Symptom } & Baseline & Intake 1 & Intake 2 & Follow-up \\
\hline Painful effort during defecation & $1.67(1.29)$ & $1.45(1.14)^{\mathrm{a}}$ & $1.15(0.97)^{\mathrm{a}}$ & $1.16(0.88)^{\mathrm{a}}$ \\
Feeling of incomplete evacuation & $2.27(1.03)$ & $2.01(1.31)^{\mathrm{a}}$ & $1.61(1.28)^{\mathrm{a}}$ & $1.53(1.32)^{\mathrm{a}}$ \\
Straining during defecation & $2.38(0.83)$ & $2.00(0.67)^{\mathrm{a}}$ & $1.75(1.19)^{\mathrm{a}}$ & $1.71(1.17)^{\mathrm{a}}$ \\
Minutes spent in lavatory per attempt & $1.90(1.06)$ & $1.68(0.90)$ & $1.68(0.80)^{\mathrm{a}}$ & $1.66(0.94)^{\mathrm{a}}$ \\
Unsuccessful defecation attempt & $1.13(0.40)$ & $1.08(0.43)$ & $1.09(0.28)$ & $1.00(0.14)^{\mathrm{a}}$ \\
Abdominal discomfort & $1.97(1.03)$ & $1.92(1.10)$ & $1.60(1.03)^{\mathrm{a}}$ & $1.30(1.08)^{\mathrm{a}}$ \\
Defecation frequency (times/wk) & $4.00(2.50)$ & $4.50(2.50)$ & $5.00(2.50)^{\mathrm{a}}$ & $5.25(2.63)^{\mathrm{a}}$ \\
Stool consistency & $3.18(1.02)$ & $3.44(1.41)^{\mathrm{a}}$ & $3.55(1.02)^{\mathrm{a}}$ & $3.19(1.21)$ \\
\hline
\end{tabular}

${ }^{a} P<0.05$, vs baseline.

Intake 1, 2-week Lactobacillus casei strain Shirota (LcS) intake; intake 2, 4-week LcS intake; follow-up, 2-week following LcS intake.

Data are presented as median (interquartile range).

Table 3. Effects of Lactobacillus casei Strain Shirota on Stool Consistency (Adapted From Chen et $\mathrm{al}^{5}$ )

\begin{tabular}{ccccc}
\hline Group & Baseline & Intake 1 & Intake 2 & Follow-up \\
\hline HS & $2.50(1.13)$ & $2.75(0.84)^{\mathrm{a}}$ & $3.04(1.30)^{\mathrm{a}}$ & $3.00(1.43)^{\mathrm{a}}$ \\
NS & $3.25(0.39)^{\mathrm{b}}$ & $3.63(1.00)$ & $3.63(0.82)$ & $3.25(1.00)$ \\
SS & $4.46(0.39)^{\mathrm{b}, \mathrm{c}}$ & $4.50(1.10)$ & $4.14(1.40)^{\mathrm{a}}$ & $4.02(1.76)^{\mathrm{a}}$ \\
\hline
\end{tabular}

${ }^{\mathrm{a} P}<0.05$, vs baseline.

${ }^{\mathrm{b}} \mathrm{P}<0.05$, vs HS.

${ }^{c} P<0.05$, vs NS.

HS, hard stool; NS, normal stool; SS, soft stool; intake 1, 2-week Lactobacillus casei strain Shirota (LcS) intake; intake 2, 4-week LcS intake; follow-up, 2-week following $\mathrm{LcS}$ intake.

Data are presented as median (interquartile range). 
Table 4. Effect of Lactobacillus casei Strain Shirota on Constipation Symptoms of Each Group (Adapted From Chen et al ${ }^{5}$ )

\begin{tabular}{|c|c|c|c|c|c|}
\hline Symptom & Group & Baseline & Intake 1 & Intake 2 & Follow-up \\
\hline \multirow[t]{3}{*}{ Painful effort during defecation } & HS & $1.75(0.99)$ & $1.14(0.88)^{\mathrm{a}}$ & $1.00(0.57)^{\mathrm{a}}$ & $1.00(0.46)^{\mathrm{a}}$ \\
\hline & NS & $1.69(1.29)$ & $1.71(1.14)^{\mathrm{a}}$ & $1.25(1.14)^{\mathrm{a}}$ & $1.29(0.9)^{\mathrm{a}}$ \\
\hline & SS & $1.44(1.30)$ & $1.86(1.35)$ & $1.66(1.63)$ & $1.69(1.20)$ \\
\hline \multirow[t]{3}{*}{ Feeling of incomplete evacuation } & HS & $2.50(1.09)$ & $2.00(0.99)^{\mathrm{a}}$ & $1.46(0.73)^{\mathrm{a}}$ & $1.22(1.12)^{\mathrm{a}}$ \\
\hline & NS & $2.20(0.84)$ & $1.86(1.33)$ & $1.50(1.72)^{c}$ & $1.36(1.50)^{\mathrm{a}}$ \\
\hline & SS & $2.37(1.31)$ & $2.55(1.21)$ & $2.23(1.04)$ & $1.73(0.75)$ \\
\hline \multirow[t]{3}{*}{ Straining during defecation } & HS & $2.76(1.00)$ & $2.00(0.62)^{\mathrm{a}}$ & $1.56(0.79)^{\mathrm{a}}$ & $1.56(0.92)^{\mathrm{a}}$ \\
\hline & NS & $2.25(0.54)^{\mathrm{b}}$ & $2.00(1.04)$ & $2.00(1.22)^{\mathrm{a}}$ & $1.90(1.10)^{\mathrm{a}}$ \\
\hline & SS & $1.97(0.9)^{b, c}$ & $2.00(0.59)$ & $1.84(1.32)$ & $1.52(1.20)^{\mathrm{a}}$ \\
\hline
\end{tabular}

${ }^{\mathrm{a}} P<0.05$, vs baseline.

${ }^{\mathrm{b}} P<0.05$, vs HS

${ }^{\mathrm{c}} \mathrm{P}<0.05$, vs NS.

HS, hard stool; NS, normal stool; SS, soft stool; intake 1, 2-week Lactobacillus casei strain Shirota (LcS) intake; intake 2, 4-week LcS intake; follow-up, 2-week following LcS intake.

Data are presented as median (interquartile range).

scale of 3.19 in follow-up, whereas, in follow-up in Table 3, those numbers seem not identical while there were scored 3 in HS $(\mathrm{n}=$ 23), 3.25 in NS ( $\mathrm{n}=27)$, and 4.02 in SS $(n=12)$. Nevertheless, it is interesting since these changes are accordingly explained by an alternation of SCFA level, furthermore, the gut microbiota adjustment.

Although the authors claimed that all constipation symptoms were significantly improved, there is some confusion in Table 4. For example, NS had more severe pain in the defection effort versus SS, and SS had less straining during defection. Clinically, constipation causes a blockage and the bowel begins to leak out watery stools around the blockage from higher up. The leak from the bowel can look like diarrhea which was always rectal tenesmus.

Lastly, this study was sponsored by Yakult. Does industry sponsorship bias research findings? Funding bias has been associated with research into chemical toxicity, tobacco, and pharmaceutical drugs. It concerns the tendency of a scientific study to support the interests of the study's financial sponsor.

Anyhow, this study suggests that probiotic beverage has an intestine-conditioning effect by improving the stool quality and rebalancing the intrinsic SCFA and Bacteroides in stool consistency.

Min Chen, ${ }^{*}$ Xujun Ye, Dan Shen, and Chunwei Ma Geriatric Department, Zhongnan Hospital, Wuhan University, China
1. Mani-López E, Palou E, López-Malo A. Probiotic viability and storage stability of yogurts and fermented milks prepared with several mixtures of lactic acid bacteria. J Dairy Sci 2014;97:2578-2590.

2. Rivière A, Selak M, Lantin D, Leroy F, De Vuyst L. Bifidobacteria and butyrate-producing colon bacteria: importance and strategies for their stimulation in the human gut. Front Microbiol 2016;7:979.

3. Jackson MI, Jewell DE. Balance of saccharolysis and proteolysis underpins improvements in stool quality induced by adding a fiber bundle containing bound polyphenols to either hydrolyzed meat or grain-rich foods. Gut Microbes 2019;10:298-320.

4. Kato-Kataoka A, Nishida K, Takada M, et al. Fermented milk containing Lactobacillus casei strain Shirota preserves the diversity of the gut microbiota and relieves abdominal dysfunction in healthy medical students exposed to academic stress. Appl Environ Microbiol 2016;82:36493658.

5. Chen S, Ou Y, Zhao L, et al. Differential effects of Lactobacillus casei strain Shirota on patients with constipation regarding stool consistency in China. J Neurogastroenterol Motil 2019;25:148-158.

6. Rome Foundation. Guidelines-Rome III diagnostic criteria for functional gastrointestinal disorders. J Gastrointestin Liver Dis 2006;15:307312 .

\section{Financial support: None.} Conflicts of interest: None. Author contributions: Min Chen: designed the study and wrote the manuscript; and Xujun Ye, Dan Shen, and Chunwei Ma: performed data analysis. 